\title{
Hopf algebras and the logarithm of the $S$-transform in free probability - Extended abstract
}

\author{
Mitja Mastnak ${ }^{1}$ and Alexandru Nica ${ }^{2}$ \\ ${ }^{1}$ Department of Mathematics and C.S., Saint Mary's University, Halifax, NS B3H 3C3, Canada \\ ${ }^{2}$ Department of Pure Mathematics, University of Waterloo, Waterloo, ON N2L 3G1, Canada
}

This document is an extended abstract of the paper 'Hopf algebras and the logarithm of the S-transform in free probability' in which we introduce a Hopf algebraic approach to the study of the operation $\nabla$ (free multiplicative convolution) from free probability.

Keywords: Hopf algebra, free probability, non-crossing partition, symmetric function

\section{Introduction}

Two basic tools of free probability are the $R$-transform and the $S$-transform. These transforms were introduced by Voiculescu in the 1980s, and are used to understand the addition and the multiplication of two free random variables respectively. The $R$-transform has a natural and very useful multi-variable extension describing the addition of two free $k$-tuples of random variables, but the problem of finding such an extension for the $S$-transform is open.

The problem of the multi-variable $S$-transform can be re-phrased as the problem of understanding the structure of the group $\left(\mathcal{G}_{k}, \otimes\right)$, where $\mathcal{G}_{k}$ is a special set of joint distributions of noncommutative $k$-tuples (see precise definition in Equation (3.3) below), and where $\bigotimes$ ("free multiplicative convolution") is a binary operation on $\mathcal{G}_{k}$ which encodes the multiplication of free $k$-tuples. At present, the structure of $\left(\mathcal{G}_{k}, \bigotimes\right)$ is well-understood only in the special case $k=1$; in this case, the $S$-transform of Voiculescu provides an isomorphism between $\mathcal{G}_{1}$ and a multiplicative group of power series in one variable. (A word of caution here: $\mathcal{G}_{1}$ is commutative, but it is easy to see that $\mathcal{G}_{k}$ is not commutative for any $k \geq 2$.)

In [4] we use Hopf algebra methods in order to study the multiplication of free $k$-tuples. Specifically, we construct a combinatorial Hopf algebra $\mathcal{Y}^{(k)}$ such that $\left(\mathcal{G}_{k}, \bigotimes\right)$ is naturally isomorphic to the the group $\mathbb{X}\left(\mathcal{Y}^{(k)}\right)$ of characters of $\mathcal{Y}^{(k)}$. We then employ the log map from characters to infinitesimal characters of $\mathcal{Y}^{(k)}$, to introduce a transform $L S_{\mu}$ for distributions $\mu \in \mathcal{G}_{k} . L S_{\mu}$ is a power series in $k$ non-commuting indeterminates $z_{1}, \ldots, z_{k}$; its coefficients can be computed from the coefficients of the $R$-transform of $\mu$

\footnotetext{
${ }^{\dagger}$ Research supported by a Discovery Grant from NSERC, Canada.

1365-8050 (c) 2009 Discrete Mathematics and Theoretical Computer Science (DMTCS), Nancy, France
} 
by using summations over chains in the lattices $N C(n)$ of non-crossing partitions. The $L S$-transform has the "linearizing" property that

$$
L S_{\mu \bowtie \nu}=L S_{\mu}+L S_{\nu}, \quad \forall \mu, \nu \in \mathcal{G}_{k} \text { such that } \mu \otimes \nu=\nu \otimes \mu .
$$

If $k=1$, then $\mathcal{Y}^{(1)}$ is naturally isomorphic to the Hopf algebra Sym of symmetric functions, and the $L S$-transform is related to the logarithm of the $S$-transform of Voiculescu, by the formula

$$
L S_{\mu}(z)=-z \log S_{\mu}(z), \quad \forall \mu \in \mathcal{G}_{1} .
$$

In this case the group $\left(\mathcal{G}_{1}, \otimes\right)$ can be identified as the group of characters of Sym, in such a way that the $S$-transform, its reciprocal $1 / S$ and its $\log$ arithm $\log S$ relate in a natural sense to the sequences of complete, elementary and power sum symmetric functions.

In [4] we connect several areas in mathematics: free probability, combinatorics of non-crossing partitions, and Hopf algebras. In this extended abstract emphasis is placed on reviewing concepts in these areas needed for understanding of our paper. This is done in Sections 2, 3, and 4. In Section 5 we define the Hopf algebra $\mathcal{Y}^{(k)}$ and describe the isomorphism $\mathcal{G}_{k} \simeq \mathbb{X}\left(\mathcal{Y}^{(k)}\right)$. In Section 6 we explain the way logarithm of character on $\mathcal{Y}^{(k)}$ gives rise to the $L S$ transform. Section 7 is then a review of what happens in the special case when $k=1$. We do not include any of the proofs.

\section{Notation: $N C(n)$ and power series}

\subsection{Non-crossing partitions}

We will use the standard conventions of notation for non-crossing partitions (as in [8], or in Lecture 9 of [6]). For a positive integer $n$, the set of all non-crossing partitions of $\{1, \ldots, n\}$ will be denoted by $N C(n)$. For $\pi \in N C(n)$, the number of blocks of $\pi$ will be denoted by $|\pi|$. On $N C(n)$ we consider the partial order given by reversed refinement: for $\pi, \rho \in N C(n)$, we write " $\pi \leq \rho$ " to mean that every block of $\rho$ is a union of blocks of $\pi$. The minimal and maximal element of $(N C(n), \leq)$ are denoted by $0_{n}$ (the partition of $\{1, \ldots, n\}$ into $n$ blocks of 1 element each) and respectively $1_{n}$ (the partition of $\{1, \ldots, n\}$ into 1 block of $n$ elements).

Every partition $\pi \in N C(n)$ has associated to it a permutation of $\{1, \ldots, n\}$, which is denoted by $P_{\pi}$, and is defined by the following prescription: for every block $B=\left\{b_{1}, \ldots, b_{m}\right\}$ of $\pi$, with $b_{1}<\cdots<b_{m}$, one creates a cycle of $P_{\pi}$ by putting

$$
P_{\pi}\left(b_{1}\right)=b_{2}, \ldots, P_{\pi}\left(b_{m-1}\right)=b_{m}, P_{\pi}\left(b_{m}\right)=b_{1} .
$$

Note that in the particular case when $\pi=0_{n}$ we have that $P_{0_{n}}$ is the identity permutation of $\{1, \ldots, n\}$, while for $\pi=1_{n}$ we have that $P_{1_{n}}$ is the cycle $1 \mapsto 2 \mapsto \cdots \mapsto n \mapsto 1$.

The Kreweras complementation map is a special order-reversing bijection $K: N C(n) \rightarrow N C(n)$. In this paper we will use its description in terms of permutations associated to non-crossing partitions: for $\pi \in N C(n)$, the Kreweras complement of $\pi$ is the partition $K(\pi) \in N C(n)$ uniquely determined by the fact that its associated permutation is

$$
P_{K(\pi)}=P_{\pi}^{-1} P_{1_{n}}
$$


Formula (2.1) can be extended in order to cover the concept of relative Kreweras complement of $\pi$ in $\rho$, for $\pi, \rho \in N C(n)$ such that $\pi \leq \rho$. This is the partition in $N C(n)$, denoted by $K_{\rho}(\pi)$, uniquely determined by the fact that the permutation associated to it is

$$
P_{K_{\rho}(\pi)}=P_{\pi}^{-1} P_{\rho}
$$

Clearly, the Kreweras complementation map $K$ from 2.1 is the relative complementation with respect to the maximal element $1_{n}$ of $N C(n)$.

The formulas [2.1, , 2.2) do not follow exactly the original approach used by Kreweras in [3], but are easily seen to be equivalent to it (see e.g. [6], Exercise 18.25 on p. 301).

\subsection{Power series and generalized coefficients}

Let $k$ be a positive integer. We use the notation $[k]^{*}$ for the set of all words of finite length over the alphabet $\{1, \ldots, k\}$ :

$$
[k]^{*}:=\bigcup_{n=0}^{\infty}\{1, \ldots, k\}^{n}
$$

The length of a word $w \in[k]^{*}$ will be denoted by $|w|$. We follow the standard procedure of including into $[k]^{*}$ a unique word $\phi$ with $|\phi|=0$.

We will use the notation $\mathbb{C}_{0}\left\langle\left\langle z_{1}, \ldots, z_{k}\right\rangle\right\rangle$ for the set of power series with complex coefficients and with vanishing constant term in the non-commuting indeterminates $z_{1}, \ldots, z_{k}$. The general form of a series $f \in \mathbb{C}_{0}\left\langle\left\langle z_{1}, \ldots, z_{k}\right\rangle\right\rangle$ is thus

$$
f\left(z_{1}, \ldots, z_{k}\right)=\sum_{n=1}^{\infty} \sum_{i_{1}, \ldots, i_{n}=1}^{k} \alpha_{\left(i_{1}, \ldots, i_{n}\right)} z_{i_{1}} \cdots z_{i_{n}}=\sum_{\substack{w \in[k]^{*} \\|w| \geq 1}} \alpha_{w} z_{w}
$$

where the coefficients $\alpha_{w}$ are from $\mathbb{C}$ and where we write in short $z_{w}:=z_{i_{1}} \cdots z_{i_{n}}$ for $w=\left(i_{1}, \ldots, i_{n}\right) \in$ $\{1, \ldots, k\}^{n}, n \geq 1$.

For every word $w \in[k]^{*}$ with $|w| \geq 1$ we will denote by

$$
\mathrm{Cf}_{w}: \mathbb{C}_{0}\left\langle\left\langle z_{1}, \ldots, z_{k}\right\rangle\right\rangle \rightarrow \mathbb{C}
$$

the linear functional which extracts the coefficient of $z_{w}$ in a series $f \in \mathbb{C}_{0}\left\langle\left\langle z_{1}, \ldots, z_{k}\right\rangle\right\rangle$. Thus for $f$ written as in Equation 2.4 we have $\mathrm{Cf}_{w}(f)=\alpha_{w}$.

Suppose we are given a positive integer $n$, a word $w=\left(i_{1}, \ldots, i_{n}\right) \in\{1, \ldots, k\}^{n}$ and a partition $\pi \in N C(n)$. We define a (generally non-linear) functional

$$
\mathrm{Cf}_{w ; \pi}: \mathbb{C}_{0}\left\langle\left\langle z_{1}, \ldots, z_{k}\right\rangle\right\rangle \rightarrow \mathbb{C},
$$

as follows. For every block $B=\left\{b_{1}, \ldots, b_{m}\right\}$ of $\pi$, with $1 \leq b_{1}<\cdots<b_{m} \leq n$, let us use the notation

$$
w\left|B=\left(i_{1}, \ldots, i_{n}\right)\right| B:=\left(i_{b_{1}}, \ldots, i_{b_{m}}\right) \in\{1, \ldots, k\}^{m} .
$$

Then we define

$$
\mathrm{Cf}_{w ; \pi}(f):=\prod_{B \text { block of } \pi} \mathrm{Cf}_{w \mid B}(f), \forall f \in \mathbb{C}_{0}\left\langle\left\langle z_{1}, \ldots, z_{k}\right\rangle\right\rangle .
$$

(For example if $w=\left(i_{1}, \ldots, i_{5}\right)$ is a word of length 5 and if $\pi=\{\{1,4,5\},\{2,3\}\} \in N C(5)$, then the above formula comes to $\mathrm{Cf}_{\left(i_{1}, i_{2}, i_{3}, i_{4}, i_{5}\right) ; \pi}(f)=\mathrm{Cf}_{\left(i_{1}, i_{4}, i_{5}\right)}(f) \cdot \mathrm{Cf}_{\left(i_{2}, i_{3}\right)}(f), f \in \mathbb{C}_{0}\left\langle\left\langle z_{1}, \ldots, z_{k}\right\rangle\right\rangle$.) 


\section{Free probability}

\subsection{Noncommutative probability space, random variables, and moments}

Let $(\mathcal{A}, \varphi)$ be a noncommutative probability space, that is $\mathcal{A}$ is a unital algebra over $\mathbb{C}$ and $\varphi: \mathcal{A} \rightarrow \mathbb{C}$ is a linear functional such that $\varphi\left(1_{\mathcal{A}}\right)=1$. We refer to the elements of $\mathcal{A}$ as random variables. Given a random variable $a \in \mathcal{A}$, we refer to the number $\varphi\left(a^{n}\right)$ by calling it the moment of order $n$ of $a$. The generating series for the moments of $a$,

$$
M_{a}(z)=\sum_{n=1}^{\infty} \varphi\left(a^{n}\right) z^{n},
$$

is called the moment series of $a$. This terminology extends to the situation when we deal with a $k$-tuple $a_{1}, \ldots, a_{k}$ of elements of $\mathcal{A}$. Then family

$$
\left\{\varphi\left(a_{i_{1}} \ldots a_{i_{n}}\right) \mid n \leq 1,1 \leq i_{1}, \ldots, i_{n} \leq k\right\}
$$

is called the family of joint moments of $a_{1}, \ldots, a_{k}$. These joint moments are the coefficients of a formal power series in $k$ noncommuting indeterminates $z_{1}, \ldots, z_{k}$, which is denoted by $M_{a_{1}, \ldots, a_{k}}$ and is called the joint moment series of $a_{1}, \ldots, a_{k}$ :

$$
M_{a_{1}, \ldots, a_{k}}\left(z_{1}, \ldots, z_{k}\right)=\sum_{n=1}^{\infty} \sum_{i_{1}, \ldots, i_{n}=1}^{k} \varphi\left(a_{i_{1}} \ldots a_{i_{n}}\right) z_{i_{1}} \ldots z_{i_{n}} .
$$

\subsection{Free independence}

We say that unital subalgebras $\mathcal{A}_{1}, \ldots, \mathcal{A}_{n}$ of $\mathcal{A}$ are freely independent if $\varphi\left(a_{1} \ldots a_{m}\right)=0$ whenever we have the following

- $a_{j} \in \mathcal{A}_{i(j)}$ for $j=1, \ldots, m$, where $i(1) \neq i(2), i(2) \neq i(3), \ldots, i(m-1) \neq i(m)$, and

- $\varphi\left(a_{j}\right)=0$ for $j=1, \ldots, m$.

We say that subsets $\mathcal{S}_{1}, \ldots, \mathcal{S}_{n}$ of $\mathcal{A}$ are freely independent if the unital subalgebras $\mathcal{A}_{i}=\left\langle\mathcal{S}_{i}\right\rangle, i=$ $1, \ldots, n$, generated by these subsets are freely independent. In particular, we say that $\left\{a_{1}, \ldots, a_{k}\right\}$ is freely independent from $\left\{b_{1}, \ldots, b_{k}\right\}$, if unital subalgebras generated by these $k$-tuples are freely independent.

\subsection{Distributions and free multiplicative convolution}

If $a_{1}, \ldots, a_{k}$ is a $k$-tuple of elements of $\mathcal{A}$, then the distribution of $\left(a_{1}, \ldots, a_{k}\right)$ is the linear functional $\mu$ on the algebra of noncommutative polynomials $\mathbb{C}\left\langle X_{1}, \ldots, X_{k}\right\rangle$ defined by

$$
\mu\left(X_{i_{1}} \cdots X_{i_{n}}\right)=\varphi\left(a_{i_{1}} \cdots a_{i_{n}}\right), \quad \forall n \geq 0, \forall 1 \leq i_{1}, \ldots, i_{n} \leq k .
$$

We denote by $\mathcal{D}_{\text {alg }}(k)$ the set of linear functionals on $\mathbb{C}\left\langle X_{1}, \ldots, X_{k}\right\rangle$ that arise in this way. Clearly, this is just the set of all linear functionals on $\mathbb{C}\left\langle X_{1}, \ldots, X_{k}\right\rangle$ such that $\mu(1)=1$. On $\mathcal{D}_{\text {alg }}(k)$ we 
have a binary operation $\nabla$ which reflects the multiplication of two freely independent $k$-tuples in a noncommutative probability space. That is, $\nabla$ is well-defined and uniquely determined by the following requirement: if $a_{1}, \ldots, a_{k}, b_{1}, \ldots, b_{k}$ are elements in a noncommutative probability space $(\mathcal{A}, \varphi)$ such that $\left(a_{1}, \ldots, a_{k}\right)$ has distribution $\mu,\left(b_{1}, \ldots, b_{k}\right)$ has distribution $\nu$, and $\left\{a_{1}, \ldots, a_{k}\right\}$ is freely independent from $\left\{b_{1}, \ldots, b_{k}\right\}$, then it follows that the distribution of $\left(a_{1} b_{1}, \ldots, a_{k} b_{k}\right)$ is equal to $\mu \otimes \nu$. The operation $\otimes$ on $\mathcal{D}_{\text {alg }}(k)$ is associative and unital, where the unit is the functional $\mu_{o} \in \mathcal{D}_{\text {alg }}(k)$ with $\mu_{o}\left(X_{i_{1}} \cdots X_{i_{n}}\right)=1$ for all $n \geq 1$ and $1 \leq i_{1}, \ldots, i_{n} \leq k$. A distribution $\mu \in \mathcal{D}_{\text {alg }}(k)$ is invertible with respect to $\otimes$ if and only if it satisfies $\mu\left(X_{i}\right) \neq 0, \forall 1 \leq i \leq k$; and moreover, the subset

$$
\mathcal{G}_{k}:=\left\{\mu \in \mathcal{D}_{\text {alg }}(k) \mid \mu\left(X_{i}\right)=1, \quad \forall 1 \leq i \leq k\right\}
$$

is a subgroup in the group of invertibles with respect to $\otimes$. For a basic introduction to free multiplicative convolution, we refer to Section 3.6 of [12] or to Lecture 14 in [6].

\subsection{R-transform}

Let $\mu$ be a distribution in $\mathcal{D}_{\text {alg }}(k)$, that is, $\mu: \mathbb{C}\left\langle X_{1}, \ldots, X_{k}\right\rangle \rightarrow \mathbb{C}$ is a linear functional such that $\mu(1)=1$. The $R$-transform of $\mu$ is the series $R_{\mu} \in \mathbb{C}_{0}\left\langle\left\langle z_{1}, \ldots, z_{k}\right\rangle\right\rangle$ uniquely determined by the requirement that for every $n \geq 1$ and every $1 \leq i_{1}, \ldots, i_{n} \leq k$ one has

$$
\mu\left(X_{i_{1}} \cdots X_{i_{n}}\right)=\sum_{\pi \in N C(n)} \mathrm{Cf}_{\left(i_{1}, \ldots, i_{n}\right) ; \pi}\left(R_{\mu}\right) .
$$

If $\mu$ is the distribution corresponding to a $k$-tuple $a_{1}, \ldots, a_{k}$, then we often write $R_{a_{1}, \ldots, a_{k}}=R_{\mu}$.

It is easy to see that Equation 3.4 does indeed determine a unique series in $\mathbb{C}_{0}\left\langle\left\langle z_{1}, \ldots, z_{k}\right\rangle\right\rangle$. The coefficients of $R_{\mu}$ are called the free cumulants of $\mu$, and because of this reason Equation (3.4) is sometimes referred to as "the moment-cumulant formula" - see Lectures 11 and 16 in [6].

An important point for the present paper is that the $R$-transform has a very nice behaviour under the operation $\otimes$. This is recorded in the next proposition.

Proposition 3.1 Let $\mu, \nu$ be distributions in $\mathcal{D}_{\mathrm{alg}}(k)$, and let $w$ be a word in $[k]^{*}$, with $|w| \geq 1$. Then

$$
C f_{w}\left(R_{\mu \bowtie \nu}\right)=\sum_{\pi \in N C(n)} C f_{w ; \pi}\left(R_{\mu}\right) \cdot C f_{w ; K(\pi)}\left(R_{\nu}\right) .
$$

For the proof of Proposition 3.1] we refer to Theorem 14.4 and Proposition 17.2 of [6].

\subsection{S-transform}

An efficient method for computing the moments of a product of two freely independent random variables is via the $S$-transform $S_{\mu}$ given by

$$
S_{\mu}(z)=\frac{1+z}{z} M_{\mu}^{<-1>}(z)=\frac{1}{z} R_{\mu}^{<-1>}(z)
$$

(the superscript " $<-1>$ " refers to the inverse under composition). In [11] Voiculescu showed that one has the equation

$$
S_{\mu \bowtie \nu}=S_{\mu} \cdot S_{\nu}
$$

(the result was phrased in terms of products of freely independent random variables $a, b$ in a noncommutative probability space $(\mathcal{A}, \varphi)$ ). 


\section{Graded connected Hopf algebras}

We will work with graded bialgebras over $\mathbb{C}$ and we will use the standard conventions for notations regarding them (as in the monograph [9], for instance). Our review here does not aim at generality, but just covers the specialized Hopf algebras used in the present paper.

\subsection{Notation}

Let $\mathcal{B}$ be a graded bialgebra over $\mathbb{C}$. The comultiplication and counit of $\mathcal{B}$ will be denoted by $\Delta$ and respectively $\varepsilon$ (or by $\Delta_{\mathcal{B}}$ and $\varepsilon_{\mathcal{B}}$ when necessary to distinguish $\mathcal{B}$ from other graded bialgebras that are considered at the same time).

For every $n \geq 0$, the vector subspace of $\mathcal{B}$ which consists of homogeneous elements of degree $n$ will be denoted by $\mathcal{B}_{n}$. We thus have a direct sum decomposition $\mathcal{B}=\oplus_{n=0}^{\infty} \mathcal{B}_{n}$ where

$$
\left\{\begin{array} { l } 
{ \mathcal { B } _ { 0 } \ni 1 _ { \mathcal { B } } \text { (the unit of } \mathcal { B } \text { ), } } \\
{ \mathcal { B } _ { m } \cdot \mathcal { B } _ { n } \subseteq \mathcal { B } _ { m + n } , \quad \forall m , n \geq 0 , }
\end{array} \text { and } \quad \left\{\begin{array}{l}
\left.\varepsilon\right|_{\mathcal{B}_{n}}=0, \forall n \geq 1 \\
\Delta\left(\mathcal{B}_{n}\right) \subseteq \oplus_{i=0}^{n} \mathcal{B}_{i} \otimes \mathcal{B}_{n-i}, \quad \forall n \geq 0
\end{array}\right.\right.
$$

If the space $\mathcal{B}_{0}$ of homogeneous elements of degree 0 is equal to $\mathbb{C}_{\mathcal{B}}$ then we say that the graded bialgebra $\mathcal{B}$ is connected.

\subsection{Convolution Algebra}

Let $\mathcal{B}$ be a graded connected bialgebra, let $\mathcal{M}$ be a unital algebra over $\mathbb{C}$, and let $L(\mathcal{B}, \mathcal{M})$ denote the vector space of all linear maps from $\mathcal{B}$ to $\mathcal{M}$. For $\xi, \eta \in L(\mathcal{B}, \mathcal{M})$ one can define their convolution product, denoted here simply as " $\xi \eta$ ", by the formula

$$
\xi \eta:=\text { Mult } \circ(\xi \otimes \eta) \circ \Delta,
$$

where Mult $: \mathcal{M} \otimes \mathcal{M} \rightarrow \mathcal{M}$ is the linear map given by multiplication (Mult $(x \otimes y)=x y$ for $x, y \in \mathcal{M}$ ).

When endowed with its usual vector space structure and with the convolution product, $L(\mathcal{B}, \mathcal{M})$ becomes itself a unital algebra over $\mathbb{C}$. The unit of $L(\mathcal{B}, \mathcal{M})$ is the linear map $\mathcal{B} \ni b \mapsto \varepsilon(b) 1_{\mathcal{M}}$, which by a slight abuse of notation is still denoted as $\varepsilon$ (same notation as for the counit of $\mathcal{B}$ ).

\subsection{Calculus in a convolution algebra}

For every $\xi \in L(\mathcal{B}, \mathcal{M})$ it makes sense to form polynomial expressions in $\xi$, that is, expressions of the form

$$
\sum_{\ell=0}^{n} t_{\ell} \xi^{\ell} \in L(\mathcal{B}, \mathcal{M}), \text { for } n \geq 0 \text { and } t_{0}, t_{1}, \ldots, t_{n} \in \mathbb{C},
$$

where the $\xi^{\ell}(0 \leq \ell \leq n)$ are convolution powers of $\xi$, and we make the convention that $\xi^{0}:=\varepsilon$ (the unit of $L(\mathcal{B}, \mathcal{M})$ ). An important point for the present paper is that if $\xi$ is such that $\xi\left(1_{\mathcal{B}}\right)=0$ then it also makes sense to define an element

$$
\eta:=\sum_{\ell=0}^{\infty} t_{\ell} \xi^{\ell} \in L(\mathcal{B}, \mathcal{M})
$$

for an arbitrary infinite sequence $\left(t_{\ell}\right)_{\ell \geq 0}$ in $\mathbb{C}$. Indeed, if $\xi\left(1_{\mathcal{B}}\right)=0$ then due to the fact that $\Delta$ respects the grading one immediately sees that $\xi^{\ell}$ vanishes on $\mathcal{B}_{n}$ whenever $\ell>n$. Thus $\eta$ from 4.2 can be 
defined as the unique linear map from $\mathcal{B}$ to $\mathcal{M}$ which satisfies

$$
\left.\eta\right|_{\mathcal{B}_{n}}=\left.\left(\sum_{\ell=0}^{N} t_{\ell} \xi^{\ell}\right)\right|_{\mathcal{B}_{n}}, \quad \forall N \geq n \geq 0 .
$$

\subsection{The antipode}

Every graded connected bialgebra $\mathcal{B}$ is in fact a Hopf algebra [5] - this means, by definition, that the identity map id $: \mathcal{B} \rightarrow \mathcal{B}$ is an invertible element in the convolution algebra $L(\mathcal{B}, \mathcal{B})$. The inverse of id is called the antipode of $\mathcal{B}$ and is denoted by $S$. A reason why $S$ is sure to exist is that one can introduce it via a series expansion as in 4.2 above, which mimics the geometric series expansion of $(\varepsilon-(\varepsilon-\mathrm{id}))^{-1}$. That is, one can put

$$
S:=\varepsilon+\sum_{\ell=1}^{\infty}(\varepsilon-\mathrm{id})^{\ell} \in L(\mathcal{B}, \mathcal{B})
$$

(which makes sense because $(\varepsilon-\mathrm{id})\left(1_{\mathcal{B}}\right)=0$ ), and can then verify that $S$ from 4.4 has indeed the property that $S$ id $=\varepsilon=$ id $S$. See Lemma 14 in [10].

\subsection{The group of characters}

A unital algebra homomorphism from $\mathcal{B}$ to $\mathbb{C}$ is called a character. The set of all characters of $\mathcal{B}$ will be denoted by $\mathbb{X}(\mathcal{B})$. It is easy to verify that the convolution product of two characters is again a character. Moreover, if $\eta$ is a character then it is obvious that the functional $\eta \circ S \in L(\mathcal{B}, \mathbb{C})$ is a character as well, and it is easy to verify that $\eta(\eta \circ S)=\varepsilon=(\eta \circ S) \eta$. Hence $\mathbb{X}(\mathcal{B})$ is a subgroup of the group of invertibles of $L(\mathcal{B}, \mathbb{C})$, and is thus referred to as the group of characters of $\mathcal{B}$.

\subsection{Exponentials and logarithms for functionals}

Let $\mathcal{B}$ be a graded connected Hopf algebra. If $\xi$ is a functional in $L(\mathcal{B}, \mathbb{C})$ such that $\xi\left(1_{\mathcal{B}}\right)=0$, then it makes sense to define its exponential by the familiar formula

$$
\exp \xi=\sum_{\ell=0}^{\infty} \frac{1}{\ell !} \xi^{\ell}
$$

It is easy to see that exp maps bijectively the set of functionals $\left\{\xi \in L(\mathcal{B}, \mathbb{C}) \mid \xi\left(1_{\mathcal{B}}\right)=0\right\}$ onto $\left\{\eta \in L(\mathcal{B}, \mathbb{C}) \mid \eta\left(1_{\mathcal{B}}\right)=1\right\}$; the inverse of this bijection is denoted as "log" and can be described by using the Taylor series expansion for logarithm:

$$
\log \eta=-\sum_{\ell=1}^{\infty} \frac{1}{\ell}(\varepsilon-\eta)^{\ell}, \quad \text { for } \eta \in L(\mathcal{B}, \mathbb{C}) \text { with } \eta\left(1_{\mathcal{B}}\right)=1 .
$$

By adjusting the familiar argument for the exponential of a sum of two matrices, one finds that

$$
\exp \left(\xi_{1}+\xi_{2}\right)=\exp \left(\xi_{1}\right) \exp \left(\xi_{2}\right), \forall \xi_{1}, \xi_{2} \in L(\mathcal{B}, \mathbb{C}) \begin{aligned}
& \text { such that } \xi_{1}\left(1_{\mathcal{B}}\right)=\xi_{2}\left(1_{\mathcal{B}}\right)=0 \\
& \text { and such that } \xi_{1} \xi_{2}=\xi_{2} \xi_{1} .
\end{aligned}
$$


As a consequence, in the opposite direction of the exp/log bijection one finds that

$$
\begin{aligned}
& \log \left(\eta_{1} \eta_{2}\right)=\log \left(\eta_{1}\right)+\log \left(\eta_{2}\right), \quad \forall \eta_{1}, \eta_{2} \in L(\mathcal{B}, \mathbb{C}) \text { such that } \eta_{1}\left(1_{\mathcal{B}}\right)=\eta_{2}\left(1_{\mathcal{B}}\right)=1 \\
& \text { and such that } \eta_{1} \eta_{2}=\eta_{2} \eta_{1} \text {. }
\end{aligned}
$$

This exp/log bijection is a special case of standard general results from Appendix A in [7].

\section{The Hopf algebra $\mathcal{Y}^{(k)}$}

Throughout this section we fix a positive integer $k$. We use the notation $\mathcal{Y}^{(k)}$ for the commutative algebra of polynomials

$$
\mathcal{Y}^{(k)}:=\mathbb{C}\left[Y_{w}\left|w \in[k]^{*},\right| w \mid \geq 2\right]
$$

In addition to that, we will also use the following conventions of notation.

Notation 5.1 $1^{o}$ For a word $w \in[k]^{*}$ such that $|w|=1$ (i.e. such that $w=(i)$ for some $1 \leq i \leq k$ ) we put $Y_{w}:=1$ (the unit of $\mathcal{Y}^{(k)}$ ).

$2^{o}$ Let $w$ be a word in $[k]^{*}$ with $|w|=n \geq 1$, and let $\pi=\left\{A_{1}, \ldots, A_{q}\right\}$ be a partition in $N C(n)$. We will denote

$$
Y_{w ; \pi}:=Y_{w_{1}} \cdots Y_{w_{q}} \in \mathcal{Y}^{(k)},
$$

where $w_{j}=w \mid A_{j}$ for $1 \leq j \leq q$ (and where the restriction $w \mid A$ of the word $w$ to a non-empty subset $A \subseteq\{1, \ldots, n\}$ is defined in the same way as in Equation 2.5).

The comultiplication and counit of $\mathcal{Y}^{(k)}$ are defined as follows.

Definition 5.2 $1^{o}$ Let $\Delta: \mathcal{Y}^{(k)} \rightarrow \mathcal{Y}^{(k)} \otimes \mathcal{Y}^{(k)}$ be the unital algebra homomorphism uniquely determined by the requirement that for every $w \in[k]^{*}$ with $|w|=n \geq 2$ we have

$$
\Delta\left(Y_{w}\right)=\sum_{\pi \in N C(n)} Y_{w ; \pi} \otimes Y_{w ; K(\pi)},
$$

where we use the conventions of notation introduced above (cf. Equation 5.2), and where $K(\pi)$ is the Kreweras complement of a partition $\pi \in N C(n)$. For example

$$
\Delta\left(Y_{i_{1} i_{2} i_{3}}\right)=Y_{i_{1} i_{2} i_{3}} \otimes 1+Y_{i_{1} i_{2}} \otimes Y_{i_{2} i_{3}}+Y_{i_{1} i_{3}} \otimes Y_{i_{1} i_{2}}+Y_{i_{2} i_{3}} \otimes Y_{i_{1} i_{3}}+1 \otimes Y_{i_{1} i_{2} i_{3}} .
$$

$2^{o}$ Let $\varepsilon: \mathcal{Y}^{(k)} \rightarrow \mathbb{C}$ be the unital algebra homomorphism uniquely determined by the requirement that

$$
\varepsilon\left(Y_{w}\right)=0, \quad \forall w \in[k]^{*} \text { with }|w| \geq 2 .
$$

On $\mathcal{Y}^{(k)}$ we will also consider a grading, which is defined such that every generator $Y_{w}$ of $\mathcal{Y}^{(k)}$ gets to be homogeneous of degree $|w|-1$. More precisely, the homogeneous subspaces $\mathcal{Y}_{n}^{(k)}$ of $\mathcal{Y}^{(k)}$ are defined as follows.

Notation 5.3 For every $n \geq 0$ we denote

$$
\mathcal{Y}_{n}^{(k)}:=\operatorname{span}\left\{\begin{array}{l|l}
Y_{w_{1}} \cdots Y_{w_{q}} & \begin{array}{l}
q \geq 1, w_{1}, \ldots, w_{q} \in[k]^{*} \text { with } \\
\left|w_{1}\right|, \ldots,\left|w_{q}\right| \geq 1 \text { and }\left|w_{1}\right|+\cdots+\left|w_{q}\right|=n+q
\end{array}
\end{array}\right\} .
$$


Proposition 5.4 With the comultiplication, counit and grading defined above, $\mathcal{Y}^{(k)}$ becomes a graded connected Hopf algebra.

We conclude this section by describing an isomorphism from $\mathcal{G}_{k}$ to the group of characters of $\mathcal{Y}^{(k)}$.

Definition 5.5 Let $\mu$ be a distribution in $\mathcal{G}_{k}$ and consider the $R$-transform $R_{\mu}$. The character of $\mathcal{Y}^{(k)}$ associated to $\mu$ is the character $\chi_{\mu} \in \mathbb{X}\left(\mathcal{Y}^{(k)}\right)$ uniquely determined by the requirement that

$$
\chi_{\mu}\left(Y_{w}\right)=\mathrm{Cf}_{w}\left(R_{\mu}\right), \quad \forall w \in[k]^{*} \text { such that }|w| \geq 2 .
$$

Theorem 5.6 The map $\mu \mapsto \chi_{\mu}$ defined above is a group isomorphism from $\left(\mathcal{G}_{k}, \bigotimes\right)$ onto the group $\mathbb{X}\left(\mathcal{Y}^{(k)}\right)$ of characters on $\mathcal{Y}^{(k)}$.

\section{The LS-transform}

The $L S$-transform $L S_{\mu}$ is defined so that it stores the information about the functional $\log \chi_{\mu}$, as follows.

Definition 6.1 Let $\mu$ be a distribution in $\mathcal{G}_{k}$. The $L S$-transform of $\mu$ is the power series

$$
L S_{\mu}\left(z_{1}, \ldots, z_{k}\right):=\sum_{\substack{w \in[k]^{*},|w| \geq 2}}\left(\left(\log \chi_{\mu}\right)\left(Y_{w}\right)\right) z_{w}
$$

where $\log \chi_{\mu}: \mathbb{X}\left(\mathcal{Y}^{(k)}\right) \rightarrow \mathbb{C}$ is as in Equation 4.8 , and where the meaning of " $z_{w}$ " is same as in Equation (2.4.

As a consequence of Equation 4.8, one obtains the following.

Theorem 6.2 Let $\mu$ and $\nu$ be distributions in $\mathcal{G}_{k}$ such that $\mu \otimes \nu=\nu \otimes \mu$. Then

$$
L S_{\mu \bowtie \nu}=L S_{\mu}+L S_{\nu}
$$

In particular, formula (6.2) always applies when one of $\mu, \nu$ is the joint distribution of a repeated $k$ tuple $(a, a, \ldots, a)$, where $a$ is a random variable in a non-commutative probability space $(\mathcal{A}, \varphi)$ (This special case is of particular relevance for the analytic framework of $C^{*}$-probability spaces, as explained in Example 5.2 of [4].)

The $L S$-transform was introduced above by using the Hopf algebra $\mathcal{Y}^{(k)}$, but it can also be described directly in combinatorial terms, by using summations over chains in lattices of non-crossing partitions. We next explain how this goes.

A chain in $N C(n)$ is an object of the form

$$
\Gamma=\left(\pi_{0}, \pi_{1}, \ldots, \pi_{\ell}\right)
$$

with $\pi_{0}, \pi_{1}, \ldots, \pi_{\ell} \in N C(n)$ such that $0_{n}=\pi_{0}<\pi_{1}<\cdots<\pi_{\ell}=1_{n}$ (and where $0_{n}$ and $1_{n}$ denote the minimal and maximal element of $N C(n)$, respectively). For a chain $\Gamma$ as in 6.3 , the number $\ell$ is called 
the length of $\Gamma$ and is denoted as $|\Gamma|$. Given a formal power series $f$ in non-commuting indeterminates $z_{1}, \ldots, z_{k}$, one has a natural way of defining some "generalized coefficients"

$$
\mathrm{Cf}_{w}^{(\Gamma)}(f):=\prod_{j=1}^{\ell} \mathrm{Cf}_{w ; K_{\pi_{j}}\left(\pi_{j-1}\right)}(f),
$$

where $\Gamma=\left(\pi_{0}, \pi_{1}, \ldots, \pi_{\ell}\right)$ is a chain in $N C(n)$. By using the generalized coefficients 6.4 , the combinatorial description of the $L S$-transform is stated as follows.

Theorem 6.3 Let $\mu$ be a distribution in $\mathcal{G}_{k}$, and let $w=\left(i_{1}, \ldots, i_{n}\right)$ be a word in $[k]^{*}$, where $n \geq 2$. Consider (as in Equation 6.1 of Definition 6.1) the coefficient $\left(\log \chi_{\mu}\right)\left(Y_{w}\right)$ of $z_{w}$ in the LS-transform of $\mu$. This coefficient can be also expressed as

$$
\left(\log \chi_{\mu}\right)\left(Y_{w}\right)=\sum_{\substack{\Gamma \text { chain } \\ \text { in } N C(n)}} \frac{(-1)^{1+|\Gamma|}}{|\Gamma|} C f_{\left(i_{1}, \ldots, i_{n}\right)}^{(\Gamma)}\left(R_{\mu}\right),
$$

where $R_{\mu}$ is the R-transform of $\mu$.

\section{Case of one variable}

In the remaining part of the paper we look at the particular case when $k=1$. In this case the notations are simplified due to the fact that words over the 1-letter alphabet $\{1\}$ are determined by their lengths. We make the convention to write simply " $Y_{n}$ " instead of $Y_{(1,1, \ldots, 1)}$ with $n$ repetitions of 1 in the index; thus Equation 5.1] is now written in the form

$$
\mathcal{Y}^{(1)}=\mathbb{C}\left[Y_{n} \mid n \geq 2\right]
$$

while Equation 6.1) defining $L S_{\mu}$ reduces to

$$
L S_{\mu}(z)=\sum_{n=2}^{\infty}\left(\left(\log \chi_{\mu}\right)\left(Y_{n}\right)\right) z^{n} .
$$

A special feature of the case $k=1$ (not holding for $k \geq 2$ ) is that the operation $\nabla$ is commutative. Hence for $k=1$ the linearization property stated in Theorem 6.2 holds for all $\mu, \nu \in \mathcal{G}_{1}$. A large part of [4] is dedicated to proving that the one-dimensional $L S$-transform is related to the $S$-transform as follows.

Theorem 7.1 For a distribution $\mu \in \mathcal{G}_{1}$, the power series $S_{\mu}$ and $L S_{\mu}$ are related by

$$
L S_{\mu}(z)=-z \log S_{\mu}(z)
$$

The proof of Theorem 7.1 is obtained by following the connections that $\mathcal{Y}^{(1)}$ has with symmetric functions. Let

$$
\text { Sym }=\mathbb{C}\left[p_{n} \mid n \in \mathbb{N}\right]=\mathbb{C}\left[e_{n} \mid n \in \mathbb{N}\right]=\mathbb{C}\left[h_{n} \mid n \in \mathbb{N}\right],
$$


be the Hopf algebra of symmetric functions, where $\left(e_{n}\right)_{n=1}^{\infty},\left(h_{n}\right)_{n=1}^{\infty}$, and $\left(p_{n}\right)_{n=1}^{\infty}$ are sequences of elementary, complete homogeneous, and power sum symmetric functions, respectively. If we use the convention that $e_{0}=h_{0}=p_{0}=1$, then the comultiplication formulas for these sequences are as follows

$$
\Delta\left(e_{n}\right)=\sum_{i=0}^{n} e_{i} \otimes e_{n-i}, \quad \Delta\left(h_{n}\right)=\sum_{i=0}^{n} h_{i} \otimes h_{n-i}, \quad \Delta\left(p_{n}\right)=p_{n} \otimes 1+1 \otimes p_{n},
$$

for all $n \geq 1$. We introduce a new sequence of symmetric functions $\left(y_{n}\right)_{n=2}^{\infty}$ as follows:

$$
y_{n}=\sum_{\substack{\pi=\left\{A_{1}, \ldots, A_{q}\right\} \\ \text { in } N C(n-1)}} e_{\left|A_{1}\right|} \cdots e_{\left|A_{q}\right|} \in \text { Sym. }
$$

Clearly, every $y_{n}$ is a homogeneous symmetric function of degree $n-1$. We also use the convention that $y_{0}=y_{1}=1$.

Theorem 7.2 The map $\Phi: \mathcal{Y}^{(1)} \rightarrow$ Sym, given by $Y_{n} \mapsto y_{n}$ is an isomorphism of graded connected Hopf algebras. Furthermore, if $\theta_{\mu}=\chi_{\mu} \circ \Phi^{-1}:$ Sym $\rightarrow \mathbb{C}$, then we have

$$
\begin{aligned}
\theta_{\mu}\left(y_{n}\right) & =C f_{n}\left(R_{\mu}\right), \\
\theta_{\mu}\left(h_{n}\right) & =(-1)^{n} C f_{n}\left(S_{\mu}\right), \\
\theta_{\mu}\left(e_{n}\right) & =C f_{n}\left(1 / S_{\mu}\right), \\
\theta_{\mu}\left(p_{n}\right) & =(-1)^{n} n C f_{n}\left(\log S_{\mu}\right) .
\end{aligned}
$$

Remark 7.3 If we define a character $\zeta: \mathcal{Y}^{(1)} \rightarrow \mathbb{C}$ by $\zeta\left(Y_{n}\right)=1$ for $n \in \mathbb{N}$, then the pair $\left(\mathcal{Y}^{(1)}, \zeta\right)$ is a combinatorial Hopf algebra in the sense of Aguiar, Bergeron and Sottile [1]. It turns out that the isomorphism $\Phi: \mathcal{Y}^{(1)} \rightarrow$ Sym above is the unique homomorphism of graded Hopf algebras satisfying $\zeta=\zeta_{\mathrm{sym}} \Phi$, which is guaranteed by Theorem 4.3 of [1].

Remark 7.4 Having placed the homomorphism $\Phi$ in the framework of combinatorial Hopf algebras leads to an interesting alternative description of the symmetric functions $\left\{y_{n} \mid n \geq 2\right\}$, as linear combinations of monomial quasi-symmetric functions. For every $m$-tuple of positive integers $\left(r_{1}, \ldots, r_{m}\right)$, the corresponding monomial quasi-symmetric function $M_{\left(r_{1}, \ldots, r_{m}\right)}$ is defined as

$$
M_{\left(r_{1}, \ldots, r_{m}\right)}=\sum_{1 \leq i_{1}<i_{2}<\cdots<i_{m}} x_{i_{1}}^{r_{1}} x_{i_{2}}^{r_{2}} \cdots x_{i_{m}}^{r_{m}}
$$

where $\left\{x_{i} \mid i \geq 1\right\}$ is a family of commuting indeterminates. Using some results of Ehrenborg [2] we can show that

$$
y_{n}=\sum_{\substack{\Gamma=\left(\pi_{o}, \pi_{1}, \ldots, \pi_{\ell}\right) \\ \text { chain in } N C(n)}} M_{\left(\left|\pi_{0}\right|-\left|\pi_{1}\right|,\left|\pi_{1}\right|-\left|\pi_{2}\right|, \ldots,\left|\pi_{\ell-1}\right|-\left|\pi_{\ell}\right|\right)}, \forall n \geq 2 .
$$


Remark 7.5 A 'direct' proof of the fact that the map $\Phi$ of Theorem 7.2 respects comultiplication would go as follows. Using recursion

$$
y_{n}=\sum_{m=2}^{n}\left(e_{m-1} \cdot \sum_{1=i_{1}<i_{2}<\cdots<i_{m}=n} y_{i_{2}-i_{1}} y_{i_{3}-i_{2}} \cdots y_{i_{m}-i_{m-1}}\right) .
$$

one has to establish that

$$
\Delta\left(y_{n}\right)=\sum_{\pi \in N C(n)} y_{\pi} \otimes y_{K(\pi)}, \quad \forall n \geq 2 .
$$

This can be done by induction, using the formula for the comultiplication of $e_{n}$ 's. However this induction argument is quite lengthy and significantly more involved then the approach we take in [4]. There we observe that for $\mu \in \mathcal{G}_{1}$ we have $\theta_{\mu} \circ \Phi=\chi_{\mu}$ and that $\theta_{\mu}\left(h_{n}\right)=(-1)^{n} \mathrm{Cf}_{n}\left(S_{\mu}\right)$ (more precisely in [4] we define $\theta$ by $\theta_{\mu}\left(h_{n}\right)=(-1)^{n} \mathrm{Cf}_{n}\left(S_{\mu}\right)$ and use relationship between the $R$-transform and the $S$ transform to establish $\left.\theta_{\mu} \circ \Phi=\chi_{\mu}\right)$. Since due to the multiplicativity of the $S$-transform we know that $\theta: \mathcal{G}_{1} \rightarrow \mathbb{X}(\mathrm{Sym}), \mu \mapsto \theta_{\mu}$ is a group isomorphism, and since due to the Cartier-Kostant-Milnor-Moore

Theorem (see e.g. Theorem 13.0.1 on p. 274 of $[\overline{9}])$ ) $\mathcal{Y}^{(1)}$ is isomorphic to Sym we then show that $\Phi$ must be a coalgebra map.

\section{References}

[1] M. Aguiar, N. Bergeron, F. Sottile. Combinatorial Hopf algebras and generalized Dehn-Somerville equations, Compositio Mathematica 142 (2006), 1-30.

[2] R. Ehrenborg. On posets and Hopf algebras, Advances in Mathematics 119 (1996), 1-25.

[3] G. Kreweras. Sur les partitions non-croisées d'un cycle, Discrete Mathematics 1 (1972), 333-350.

[4] M. Mastnak, A. Nica. Hopf algebras and the logarithm of the S-transform in free probability, to appear in Transactions of AMS, preprint, arXiv:0807.4169v1, 39pp.

[5] J.W. Milnor, J.C. Moore. On the structure of Hopf algebras, Annals of Mathematics 81 (1965), 211-264.

[6] A. Nica, R. Speicher. Lectures on the combinatorics of free probability, London Mathematical Society Lecture Note Series 335, Cambridge University Press, 2006.

[7] D. Quillen, Rational homotopy theory, Annals of Mathematics 90 (1969), 205-295.

[8] R. Simion. Noncrossing partitions, Discrete Mathematics 217 (2000), 367-409.

[9] M.E. Sweedler. Hopf algebras, Benjamin, 1969.

[10] M. Takeuchi. Free Hopf algebras generated by coalgebras, Journal of the Mathematical Society of Japan 23 (1971), 561-582.

[11] D. Voiculescu. Multiplication of certain noncommuting random variables, Journal of Operator Theory 18 (1987), 223-235.

[12] D.V. Voiculescu, K.J. Dykema, A. Nica. Free random variables, CRM Monograph Series 1, American Mathematical Society, 1992. 